\title{
Transcriptional analysis of cell wall and cuticle related genes during fruit development of two sweet cherry cultivars with contrasting levels of cracking tolerance
}

\author{
Cristián Balbontín ${ }^{1 *}$, Héctor Ayala ${ }^{1}$, Joselyn Rubilar ${ }^{1}$, Jessica Cote $^{2}$, and Carlos R. Figueroa ${ }^{3}$
}

\begin{abstract}
Rain-induced cracking before harvest is the major cause of crop loss in sweet cherry (Prunus avium [L.] L.) In order to better understand the relationship between cherry fruit cracking and gene expression, the transcriptional patterns of six genes related to cell wall modification and cuticular wax biosynthesis were analyzed during fruit setting (FS), fruit color change (FC) and fruit ripening (FR), employing two contrasting cultivars: the cracking resistant 'Kordia' and the cracking susceptible 'Bing'. The transcription levels of AP2/EREBP-type transcription factor (PaWINB), wax synthase (WS), $\beta$-ketoacyl-CoA synthase (PaKCSO), and $\beta$-galactosidase $(\beta$-Gal) showed higher levels in 'Kordia' than in 'Bing' during the FS stage, while similar values were observed in both cultivars at FR stage. In contrast to that pattern, transcription levels of expansin ( $P a E X P 1)$ were higher at FR stage in 'Kordia' than in 'Bing'. Transcript profile of lipid transport protein gene (PaLTPG1) decreased during fruit development, with higher levels in 'Bing' than in 'Kordia' at FC and FR stages suggesting no relation with cracking tolerance. The expression profiles of $P a W I N B, W S, P a K C S 6$, and $\beta$-Gal suggest that they are genes involved in conferring cracking tolerance, likely due to their function in cuticle deposition during early stages of fruit development. In addition, a greater expression level of expansin gene would allow for a faster growth rate in 'Kordia' at FR stage.
\end{abstract}

Key words: Cell wall and cuticle modification, cracking index, gene expression analysis, Prunus avium.

\section{INTRODUCTION}

Rain-induced cracking causes great economic loss in many cherry-producing areas of the world. The severity of this complex phenomenon has been associated with several physiological factors such as growing conditions, irrigation management, rootstock, fruit size, and flesh osmotic potential, however none of these factors have shown a consistent correlation with different cultivars or handling conditions (Simon, 2006; Balbontín et al., 2013). Even when there is no one cultivar completely tolerant to this problem, cultivar differences in cracking susceptibility are considerable (Christensen, 2000). Cracking index is one of the most common laboratory tests that have been used to assess the degree of tolerance among cultivars avoiding the effect of environmental conditions (Christensen, 1972; Lane et al., 2000; Moing et al., 2004). Although differences in cracking tolerance

${ }^{1}$ Universidad de Concepción, Facultad de Agronomía, Casilla 537, Chillán, Chile. "Corresponding author (cbalbontin@udec.cl).

${ }^{2}$ Universidad del Bío-Bío, Facultad de Ciencias, Casilla 447, Chillán, Chile.

${ }^{3}$ Universidad de Concepción, Facultad de Ciencias Forestales, Casilla 160-C, Concepción, Chile.

Received: 23 September 2013.

Accepted: 4 February 2014.

doi:10.4067/S0718-58392014000200006 levels have not been fully explained, a recent approach suggests that these could be related to the dynamics of the stages of fruit growth, with the properties of the cuticle being some of the most important factors involved in the onset of this problem (Knoche et al., 2004).

Sweet cherry (Prunus avium [L.] L.) fruit presents a characteristic double-sigmoid pattern of fruit growth with three distinctive growth phases: the first phase is characterized by the rapid division of mesocarp cells and implies a high expansion rate. The second phase involves the embryo development process and endocarp hardening, with a slow fruit growth. In the third phase, fruit growth restarts, the fruits change their color and the final fruit size is reached. Moreover, there are clear differences among sweet cherry cultivars regarding the dynamics of fruit growth patterns. Yamaguchi et al. (2003) have reported that a rapid enlargement of the mesocarp could induce the development of cracking in $P$. avium fruit, showing that cultivars tolerant to cracking have longer periods of cell division. The above could involve a slower fruit expansion phase and a more continuous process of synthesis and deposition of cuticular membrane components in the tolerant cultivars, preventing the emergence of cuticular micro cracks and cracking development (Bastias et al., 2010; Alkio et al., 2012).

The cuticle integrity during fruit growth has been proposed as one of the factors that could influence the 
development of fruit cracking. It is generally accepted that the cutin matrix contributes to the mechanical strength of the cuticle (Kunst and Samuels, 2003). In species with low levels of fruit cracking such as apple and tomato, increased surface area of the fruit during development is accompanied by a continuous synthesis of the components of cuticle membrane (Knoche et al., 2011). However, during active phases of growth in sweet cherry fruit, the amount of cuticular membranes per unit area decreases, resulting in the formation of microscopic cracks in the cuticle and thus increasing their sensitivity to fruit cracking (Peschel and Knoche, 2005).

Cuticle structure consists of a matrix of cutin polymers and cuticular waxes. Mutants in Arabidopsis thaliana and other species such as barley, tomato, and maize with alteration in the production of waxes have helped to elucidate different steps and genes involved in the pathway of wax production (Jenks et al., 2002; Vogg et al., 2004). Cutin monomers are synthesized from fatty acyl-CoAs by multiple hydroxylation and epoxidation reactions (Kunst and Samuels, 2003). Major classes of aliphatic cuticular wax component include alkanes, ketones, fatty alcohols, secondary alcohols, aldehydes, fatty acids, and esters produced by acyl reduction and decarbonylation reactions of very long chain fatty acids (VLCFA) in the epidermal cells (Peschel et al., 2007). The last step in the acyl reduction pathway is the synthesis of wax esters by a fatty acyl-CoA: fatty alcohol acyltransferase (wax synthase, WS). In P. avium fruit, genes involved in biosynthesis of VLCFA waxes (PaKCS6), export of cuticular lipids (PaLTPG1), and transcriptional regulation (PaWINB) are activated during fruit growth phases (Alkio et al., 2012). However, the possible involvement of these genes in the development of cherry fruit cracking in different cultivars has not been specifically elucidated to date.

Cell wall modification during fruit development is another factor related to the fruit cracking process and is a result of a combined action of numerous enzymes such as polygalacturonase, pectin methylesterase, expansin and $\beta$-galactosidase (Brummell and Harpster, 2001). $\beta$-Galactosidase ( $\beta$-Gal) is the only enzyme identified in higher plants capable of directly cleaving $\beta-(1,3$, and 4$)$ galactan bonds, contributing to cell wall degradation and extension and thus being able to play a role in cracking tolerance among cultivars. In tomato the antisense suppression of a $\beta$-galactosidase gene (TBG6) produced fruits with a substantial increase in the levels of cracking (Moctezuma et al., 2003). Similarly, it has been shown that other proteins like expansins contribute to the extension of cell walls.

Expansins are non-enzymatic proteins involved in cell wall extension in a $\mathrm{pH}$ dependent manner and consequently can be considered regulators of the elongation of plant cells. Expansins act by breaking down the links between cellulose microfibrils and xyloglucans molecules, allowing relaxation and expansion of the cell wall under tensile stress (Cosgrove, 2000). Although knowledge about the role of expansin proteins in cherry fruit cracking is still limited, in other species such as apple (Kasai et al., 2008) and litchi (Wang et al., 2006) the levels of transcription of genes coding for expansin have been correlated with this phenomenon.

Taken together, the information provided above suggests that expression levels of cell wall- and cuticlerelated genes during fruit development can provide a first approach to understanding molecular mechanisms involved in cracking tolerance in P. avium fruit. Therefore, the objective of this work was to examine the relationship between the cracking degree and the transcriptional profiles of several genes related to the cuticular wax biosynthesis and cell wall modification of two contrasting cultivars during fruit development.

\section{MATERIALS AND METHODS}

\section{Plant material}

Three 10 yr-old 'Kordia' (cracking-resistant cultivar) and 'Bing' (cracking-susceptible cultivar) sweet cherry trees grafted on 'Gisela 6' rootstock were chosen for this experiment. Trees were grown at 'El Nogal' Experimental Station at the Universidad de Concepción $\left(36^{\circ} 35^{\prime} \mathrm{S}\right.$, 72 $04^{\prime}$ W; 140 m a.s.1.), Chillán, Chile. Fruits of both cultivars were collected weekly during the 2011-2012 growing season from anthesis to ripening considering the days after full bloom (DAFB) and growing degreehours (GDH) accumulation. On each sampling date the equatorial diameter of 15 intact fruits of similar size was measured. Temperature data were obtained from an agrometeorological station located $100 \mathrm{~m}$ from the experimental plot. A base temperature of $4{ }^{\circ} \mathrm{C}$ was used according to the threshold reported by Zavalloni et al. (2006) for sour cherry (Prunus cerasus L.) vegetative growth.Fruit developmental stages were defined according to Baggiolini (1952) in fruit setting (FS), fruit color change (FC), and fruit ripening (FR). At FR, a minimum value of $18{ }^{\circ}$ Brix (total soluble solid concentration) was considered for sampling, using a RHB-32ATC optical hand held refractometer (Cole-Parmer Instruments, Vernon Hills, Illinois, USA).

\section{Determination of cracking index}

'Bing' and 'Kordia' sweet cherry fruits were carefully harvested and classified in FS, FC, and FR developmental stages. Fruits were sorted to remove any damaged fruit and a set of 15 fruits with stems attached from each biological sample (tree) were placed in distilled water at $20^{\circ} \mathrm{C}$ for $5 \mathrm{~h}$. Numbers of cracked fruits were counted at $1 \mathrm{~h}$ intervals. The cracking index $(\mathrm{CI})$ was calculated by the method reported by Bastias et al. (2010) based in the formula develop by Christensen (1972).

$$
\mathrm{CI}=\left((5 \mathrm{a}+4 \mathrm{~b}+3 \mathrm{c}+2 \mathrm{~d}+1 \mathrm{e})(\mathrm{MPV})^{-1}\right) 100
$$

where $\mathrm{a}, \mathrm{b}, \mathrm{c}, \mathrm{d}$, and e represent the number of cracked 
fruit at 1, 2, 3, 4 and $5 \mathrm{~h}$ respectively, MPV is maximum possible value $(15$ fruits $\times 5 \mathrm{~h})$.

\section{RNA isolation and cDNA synthesis}

Frozen enriched fruit exocarp fractions of FS, FC, and FR stages were used from each cultivar. Total RNA extraction was performed using a set of five fruits from each biological sample (tree) using the CTAB method (Chang et al., 1993). Isolated RNA was treated with TURBO DNAse I (Ambion, Life Technologies, Grand Island, New York, USA) and subsequently quantified using the Qubit RNA assay kit and the Qubit fluorometer (Life Technologies, USA). cDNA was synthesized from $1 \mu \mathrm{g}$ of total RNA using the RevertAid H Minus First Strand cDNA Synthesis Kit (Thermo Fisher Scientific, Waltham, Massachusetts, USA) according to the manufacturer's instructions.

\section{Genes analyzed and quantitative real-time PCR (RT- qPCR) analysis}

The transcriptional profile of six genes related to cuticular membrane biosynthesis and cell wall modification during fruit development was analyzed by quantitative real-time PCR (RT-qPCR). Four target genes of P. avium were selected from the National Center for Biotechnology Information (NCBI) database: expansin, PaEXP1 (GenBank AF297521); $\beta$-ketoacyl-CoA synthase, PaKCS6 (GenBank JU090727); AP2/EREBP-type transcription factor, PaWINB (GenBank JU090719); and lipid transport protein LTPG1, PaLTPG1 (GenBank JU09072). Two sequences of genes corresponding to $\beta$-galactosidase $(\beta$-Gal) and wax synthase $(W S)$ were retrieved from a cherry fruit EST library. Three additional genes of $P$. avium were used as reference housekeeping genes: actin (CACT1, GenBank FJ560908), protein phosphatase 2A-2 (PaPP2A, GenBank JU090712) and signal recognition particle SRP19 (PaSRP19, GenBank JU090733). Specific primer sequences for $\beta$-Gal, WS, PaEXP1 and CACT1 genes were designed using Primer 3 software (Rozen and Skaletsky, 2000) whereas amplification of PaKCS6, PaWINB, PaLTPG1, PaPP2A, and $P a S R P 19$ genes was carried out based on sequences described by Alkio et al. (2012).

Efficiencies of PCR amplification were determined from eight-fold serial dilution series with a minimum of four data points per standard curve. Primer sequences, annealing temperatures (Ta), amplicon size and efficiencies are shown in Table 1. The amplification reactions were performed using the SensiMix SYBR HiROX Kit (Bioline, Luckenwalde, Germany) according to manufacturer's instructions in an Eco Real-Time PCR System (Illumina, San Diego, California, USA). The PCR conditions were as follows: $95^{\circ} \mathrm{C}$ for $10 \mathrm{~min}$; 40 cycles of $95{ }^{\circ} \mathrm{C}$ for $15 \mathrm{~s}$, Ta for $15 \mathrm{~s}$, and $72{ }^{\circ} \mathrm{C}$ for $15 \mathrm{~s}$; and a melting curve of $95{ }^{\circ} \mathrm{C}$ for $15 \mathrm{~s}, 55^{\circ} \mathrm{C}$ for $1 \mathrm{~min}$, and $95^{\circ} \mathrm{C}$ for $15 \mathrm{~s}$. Each reaction was performed in triplicate and a negative water control was included in each run. An inter-run calibrator consisting of pooled aliquots of cDNA from FS samples of 'Bing' was included in each 48-well plate to enable calculation of relative transcript levels in separate runs.

\section{Statistical analysis}

Experiments were conducted using a completely randomized design; the main factors were cultivar ('Bing' and 'Kordia') and fruit developmental stage (FS, FC, and FR). Values of transcription levels were analyzed by ANOVA using InfoStat software, and differences were considered statistically significant according to honestly significant difference (HSD test) at $\mathrm{P} \leq 0.05$.

\section{RESULTS}

\section{Length of fruit developmental stages of sweet cherry cultivars}

In the susceptible cracking 'Bing' full bloom stage started with 314 GDH while tolerant 'Kordia' required 366 GDH to achieve the same stage. Fruit setting occurred 13 DAFB (447 GDH) in 'Bing' and 14 DAFB (502 GDH) in 'Kordia'. The first stage of fruit growth (FS) in 'Bing' was extended for $136 \mathrm{GDH}$ (21 DAFB) while in 'Kordia' this stage reached a total of $210 \mathrm{GDH}$ (35 DAFB); the onset of color change occurred with 720 units (42 DAFB) and 963 units (54 DAFB), respectively. From this stage until ripening 'Bing' accumulated $153 \mathrm{GDH}$ (63 DAFB), while 'Kordia' gained 142 GDH (66 DAFB) (Figure 1).

\section{Analysis of cracking susceptibility among cultivars at different fruit development stages}

Cracking levels increased in both cultivars during fruit

Table 1. Primer sequences $\left(5^{\prime}->3^{\prime}\right)$ used for quantitative real-time PCR (RT-qPCR) analysis of the cell wall and cuticle related genes.

\begin{tabular}{|c|c|c|c|c|c|}
\hline Gene name & Forward primer & Reverse primer & $\mathrm{Ta}$ & Efficiency & Amplicon size \\
\hline & & & ${ }^{\circ} \mathrm{C}$ & $\%$ & bp \\
\hline PaEXP1 & GCTCAGTCCAACGATAATGG & GTAACAGGCACAATCCCAG & 60 & 99 & 113 \\
\hline$\beta$-Gal & ATGCCAAGGTTTCTGTGGAG & GCTGTACGGGACCAGTGATT & 60 & 100 & 103 \\
\hline PaWINB & AATGGTGGGATTGATTGAGG & TTGTTGGTGAAGGAATTGGA & 60 & 98 & 119 \\
\hline PaKCS6 & AGGAAGAGCAACTGCTCCGA & TCTGATGGCGATCGCAGGAG & 62 & 100 & 90 \\
\hline$W S$ & TCTCAACCACCAAACACAGC & CCTTCATAGTGTCGGCGATT & 60 & 99 & 102 \\
\hline PaLTPG1 & ATGAGGAAGATGTTGGTGGTG & ACTGGTTGCTACACTTCTG & 60 & 100 & 97 \\
\hline PaSRP19 & GGTTGTAATGGCTGGATTATATGG & GAAGGGAGACGAATAAGTGTAGAG & 62 & 100 & 198 \\
\hline $\mathrm{PaPP} 2 \mathrm{~A}-2$ & CССТCACAGCCCTTATTG & ATCGCACATTGGTCCTTC & 59 & 100 & 131 \\
\hline Actin & TGAAGATTAAGGTTGTGGCTC & CGTACTCACCCTTGGAAATC & 60 & 99 & 114 \\
\hline
\end{tabular}




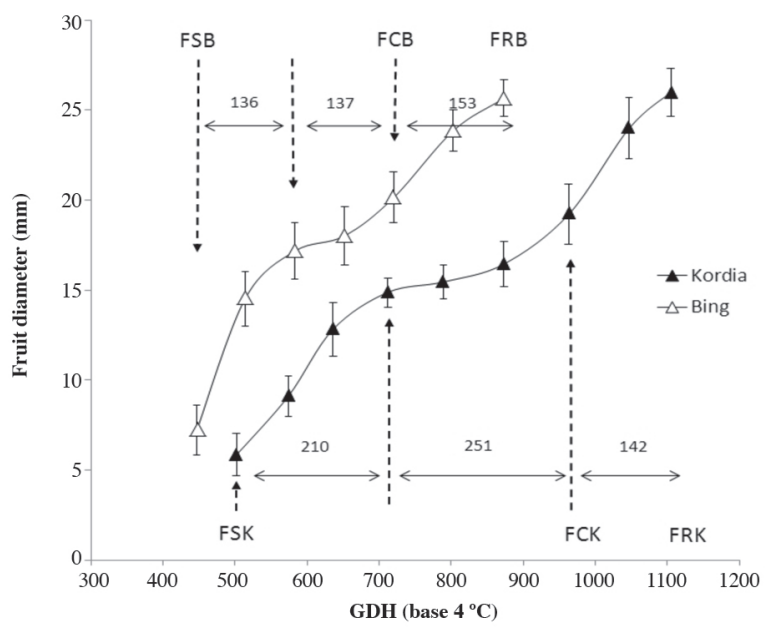

FS: fruit setting; FC: fruit color change; FR: fruit ripening; B: 'Bing'; K: 'Kordia'.

Data represent the mean \pm SD of 15 fruits.

Figure 1. Evolution of fruit growth (as fruit diameter) of 'Bing' and 'Kordia' cherry fruits during fruit development according to growing degree-hours (GDH) accumulation. Different fruit developmental stages and their lengths are represented in each fruit growth curve by dotted arrows.

development (Figure 2). At FS stage the CI was very low and no differences were found between cultivars, however significant differences were detected in FC and FR stages. At FC stage, 'Bing' showed a two-fold increase in CI compared to 'Kordia' ( $7.5 \%$ and $3.4 \%$, respectively). The highest CI was observed for 'Bing' at FR stage (45\%), whereas 'Kordia' showed an $11 \%$ of CI (Figure 2).

\section{Transcriptional analysis of genes involved in cell wall modification}

The expression patterns of PaEXPl and $\beta$-Gal were determined by RT-qPCR during fruit development of two contrasting cracking tolerance cultivars (Figures 3A, 3B). The expression level of PaEXP1 increased through fruit development in both cultivars. Nevertheless, the expression of PaEXPl was higher in 'Bing' at FC stage, whereas the expression was higher in 'Kordia' at FR stage (Figure 3A). In contrast, the transcription pattern of $\beta$-Gal differs significantly between cultivars. In 'Kordia', transcript levels of $\beta$-Gal were comparatively higher than those recorded in 'Bing' at FS and FC stages (Figure 3B). However, at FR stage the expression level of $\beta$-Gal decreased and it was similar in both cultivars.

\section{Transcriptional analysis of genes involved in cuticular wax biosynthesis}

Among the many genes involved in cuticle formation, this work focused on the study of genes previously described by Alkio et al. (2012), which have a direct relationship to the activation, synthesis and transport of cuticular wax constituents including PaWINB transcriptional factor, PaKCS6 related to the production of VLCFA, WS involved in the formation of alkyl esters, and PaLTPG1 associated with the apoplastic wax transport.

In the present study, transcript levels of $P a W I N B$ were high in both cultivars at FS stage, although levels found in 'Kordia' were significantly higher than those found in 'Bing' (Figure 3C). In both cultivars the expression of $P a W I N B$ decreased at FC and FR stages. No differences were found between cultivars in these stages.

PaKCS6 expression exhibited the highest value in 'Kordia' at FS stage, and then decreased continuously at FC and FR stages (Figure 3D) In contrast, transcript levels of PaKCS6 remained constant during early growth stages of 'Bing' and then declined during fruit ripening. At FS and FC stages, transcript levels of PaKCS6 were higher in 'Kordia' than in 'Bing'. Conversely, at FR stage both cultivars showed similar transcript levels.

In 'Kordia', transcript accumulation of WS at FS and FC stages was significantly higher than those reached by 'Bing', although the accumulation in 'Kordia' decreased at FR stage, becoming equal to those observed in 'Bing' (Figure 3E). On the other hand, transcript levels of PaLTPG1 decreased from FS to the next stages in both cultivars (Figure 3F). However, transcript levels of PaLTPG1 displayed by the susceptible cultivar are higher than those found in the resistant cultivar at FC and FR stages.

\section{DISCUSSION}

Recent studies have established that the cuticular membrane of fleshy fruits is often subjected to considerable stress. Examples include Prunus species and Vitis vinifera L. (Knoche et al., 2004; Becker and Knoche, 2012). As mentioned above, during cherry fruit growth there are two phases that involve a significant increase in

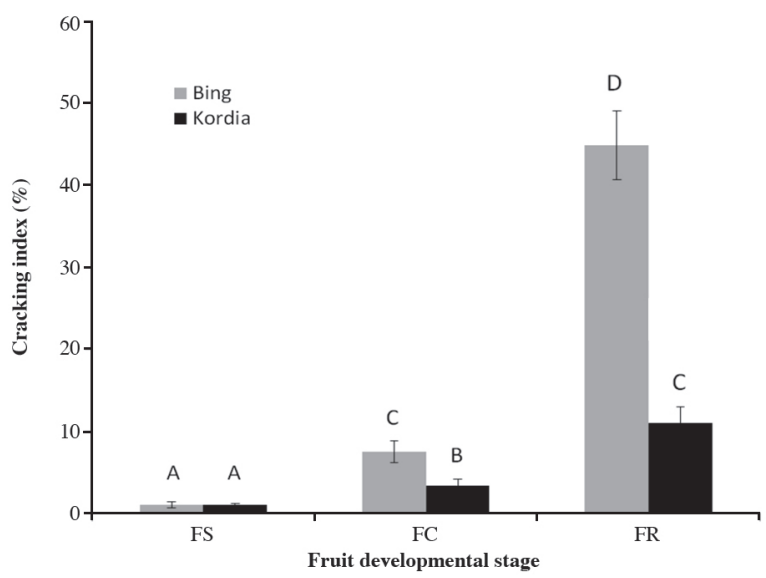

Different letters indicate significant differences between cultivars and fruit developmental stages according to HSD test $(\mathrm{P} \leq 0.05)$.

FS: fruit setting; FC: fruit color change; FR: fruit ripening.

Figure 2. Changes in cracking index (CI) during fruit development of 'Bing' and 'Kordia' cherry fruits. Data represent the mean of three replicates of 15 fruits each from each biological sample (tree) \pm SD. 

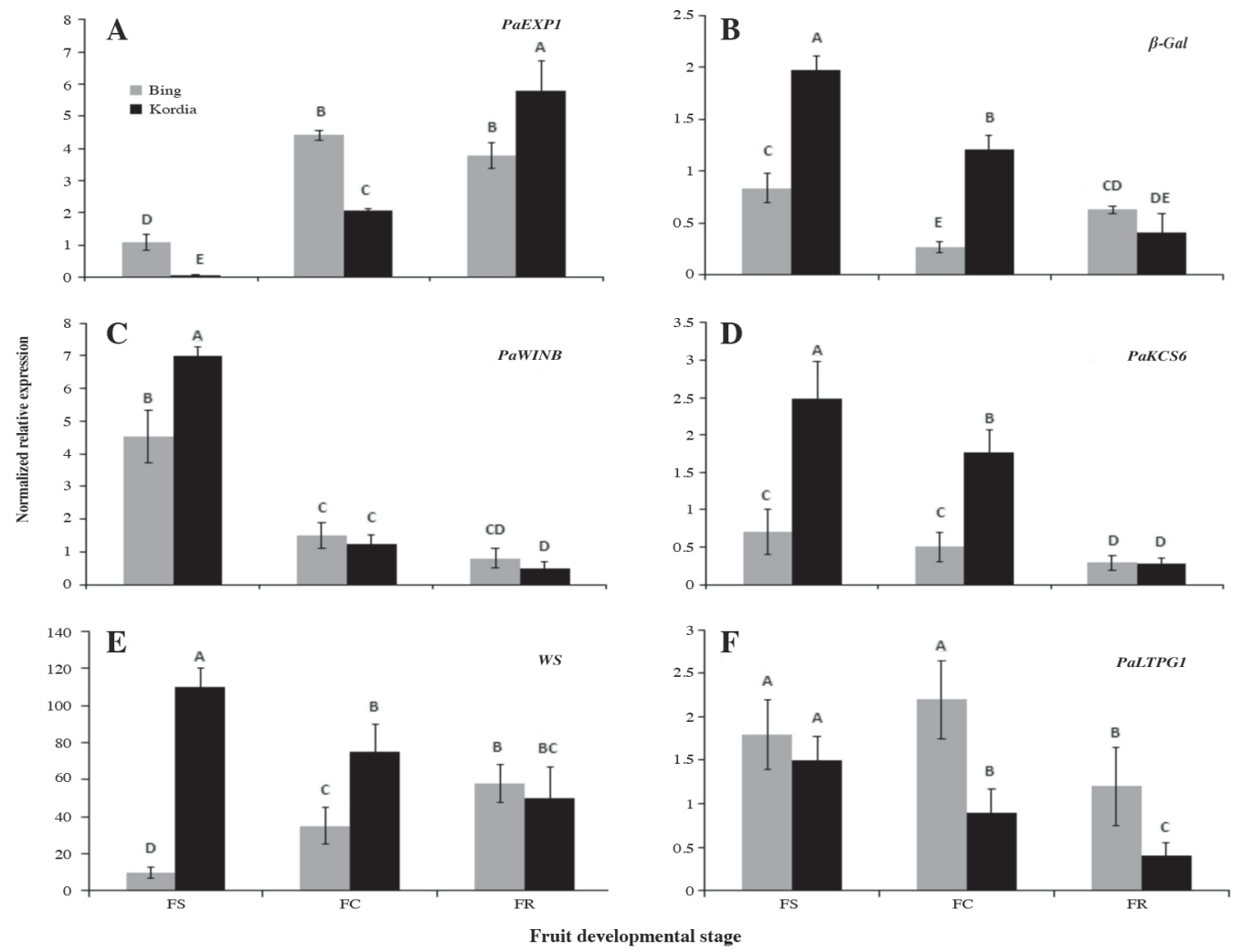

Different letters indicate significant differences between cultivars and fruit developmental stages according to HSD test $(\mathrm{P} \leq 0.05)$.

FS: fruit setting; FC: fruit color change; FR: fruit ripening.

Figure 3. Transcriptional profiles of genes involved in cell wall modification (PaEXP1 and $\beta$-Gal) and cuticular waxes (PaWINB, PaKCS6, $W S$, and PaLTPG1) during fruit development in two cultivars of sweet cherry with contrasting levels of cracking susceptibility measured by quantitative real-time PCR (RT-qPCR). The expression data correspond to the means \pm SD of three replicates expressed in arbitrary units.

fruit size and therefore expansion of the cuticle. In this study, results show a clear difference in the length of the phenological fruit stage between both cultivars. 'Bing' exhibited a short period of thermal time during the first stage of fruit growth (Figure 1), whereas 'Kordia' showed a longer period both in the first and second phase of fruit development, which results in a shorter period of final cell expansion. These differences in the length of fruit growth phases can influence the strain exerted on the cuticle of the fruit, facilitating the cracking development in "Bing, which can be seen from the FC stage until fruit ripening (Figure 2). These observations are consistent with results previously obtained by Yamaguchi et al. (2003), who suggest that a later and shorter cell expansion phase could determine a lower induction of fruit cracking.

Several authors have reported a close relationship between increased activity of expansins and fruit growth in several species. Transcripts of different genes coding for expansins accumulate during growth and ripening of pear (Hiwasa et al., 2003), apricot (Mbéguié-A-Mbéguié et al., 2002), and sour cherry fruits (Karaaslan and Hrazdina, 2010). In apple fruit, six expansin genes were identified and their expression patterns were studied during fruit growth.
Among them, MdEXPA3 is mainly expressed during fruit enlargement phase (Wakasa et al., 2003) and its high expression level in pericarp tissues has been associated with reduced level of fruit cracking (Kasai et al., 2008). In addition, an expansin gene, LcExpl, has been identified in litchi (Litchi chinensis Sonn.) and their transcript levels in pericarp tissues have shown differences between litchi cultivars with contrasting levels of cracking tolerance (Wang et al., 2006). LcExpl was detected in the pericarp at the beginning of fruit growth phase in the susceptible cultivar. In contrast, in the tolerant cultivar a high mRNA level was found at the end of this period (Wang et al., 2006). Similarly, in our study, PaEXPl showed an increase in its expression levels during the fruit stages of rapid growth in both cultivars, with the higher levels in 'Bing' than in 'Kordia' in the FS and FC stages (Figure 3A) correlating directly with $\mathrm{CI}$ observed in 'Bing' at FC stage (Figure 1). Moreover, in 'Kordia' the highest transcript accumulation of this gene is observed in the FR stage (Figure 3A) when a significant cuticle membrane strain was reported in cherry fruit (Knoche and Peschel, 2006) and a lower CI recorded in the present research (Figure 2). However, although PaEXPl transcript levels 
are higher in 'Kordia' at this stage and could help to relax the strain, our results suggest that the action of this gene alone is not sufficient to explain cracking tolerance levels, considering that 'Bing' at FC stage showed a high CI despite having a higher expression of this gene.

Regarding $\beta$-Gal, this enzyme is most active in the later stages of fruit ripening as it has been reported in tomato (Smith et al., 2002), apple (Peña and Carpita, 2004), peach (Lee et al., 2003), and mango (Prasanna et al., 2005). However, in sour cherry Kovács et al. (2008) showed that the maximum $\beta$-Gal activity occurs in the early stages of active growth and then decreases abruptly during fruit ripening, which agrees with the transcriptional profiles of $\beta$-Gal shown in our study (Figure 3B).

The activity of $\beta$-Gal has been proposed as a way to release energy stored for a period of rapid growth (Smith et al., 2002). As discussed above, the fruit growth dynamics of 'Kordia' present a later period of cell expansion in the last phase of growth stage III, providing a broader and more complete phase of cell division in the previous stages (Figure 1). In this sense, expression levels of $\beta$-Gal are higher at FS and FC stages in 'Kordia', suggesting that $\beta$-Gal enzyme could provide a greater relaxation of the walls of epidermal cells of the fruit throughout the growing period in this cultivar, decreasing its cracking susceptibility in both FC and FR stages.

$P a W I N B$ is homologous to $A$. thaliana transcription factor AP2/EREBP-type INDUCER1/SHINE1 WAX (WIN1/SHN1), whose overexpression results in increased levels of cuticular waxes and cutin (Aharoni et al., 2004). Although expression levels of PaWINB are higher in 'Kordia' only in the initial stage of fruit growth development (Figure 3C), its action may influence a subsequent activity of other genes related to the synthesis of cuticular waxes such as WS and PaCER6. In addition, SHN transcription factors can affect the expression of various cell wall-modifying genes, increasing the amount of cellulose and simultaneously decreasing lignin levels, suggesting a close coordination between the cuticle and the synthesis of cell wall polysaccharides (Shi et al., 2011; Ambavaram et al., 2011). This might suggest that the tolerant 'Kordia', besides presenting a higher gene expression related to the synthesis of cuticular waxes may also have a higher activity of cell wall modification enzymes which could provide greater exocarp flexibility preventing the appearance of micro fractures during the expansion phases of fruit development.

The 3-ketoacyl-CoA synthase (KCS) that catalyzes the biosynthesis of very long chain fatty acids (VLCFA), an important step in cuticular wax metabolism, is coded by the CER6 gene. The relationship between fruit cracking and cuticle properties has been observed in tomato fruit, where the mutation of CER6 gene leads to alteration of the cuticular wax composition, increasing water permeability and fruit cracking (Vogg et al., 2004). On the other hand, the WS enzyme is involved in the decarbonylation pathway of wax biosynthesis, leading to condensation of primary alcohols and fatty acids to produce alkyl esters. WSD1 gene mutation results in a severe reduction of waxes in the epidermis of stems in A. thaliana (Li et al., 2008). In the present study, PaKCS6 and WS exhibited a higher expression level during FS and FC stages in the tolerant 'Kordia' than that observed in the susceptible 'Bing' (Figure 3D-E). Similar results were observed by Alkio et al. (2012) in 'Regina', a sweet cherry cultivar tolerant to cracking, who reported high transcript levels of PaWINB and PaKCS6 genes in the first two stages of fruit growth development. Therefore, the up-regulation of these genes could allow for the maintenance of a more stable cuticle membrane, avoiding the emergence of microfractures in the stages of rapid fruit growth, and thus explaining lower CI values observed in 'Kordia' (Figures 3 and 2).

PaLTPG1 may play a role in apoplastic wax transport as suggested by Alkio et al. (2012). In Arabidopsis, glycosylphosphatidylinositol (GPI)-anchored lipid transfer proteins (LTPs) have been proposed as enablers in the transport of hydrophobic cuticle precursors through the hydrophilic environment of the polysaccharide cell wall to the cuticle (Yeats and Rose, 2008). However, it has been reported that in some molecules such as the dihydroxyacyl cutin precursor 2-MHG, the glycerol moiety confers sufficient polarity to allow aqueous solubility, suggesting that the participation of lipidbinding proteins may not be necessary to facilitate the transport of this major precursor of cutin biosynthesis (Yeats et al., 2012). Moreover, the presence of other carriers might be involved in this process. This may explain in part the lower transcript levels of this gene observed in 'Kordia' during FC and FR stages (Figure $3 \mathrm{~F})$. According to the transcriptional profile of PaLTPG1, it seems that this gene has a role in the early stages of fruit growth (FS and FC stages) in both cultivars (Figure $3 \mathrm{~F})$, similar to the other cuticle-related genes, since higher mRNA levels were observed in those stages compared to the FR stage. Nevertheless, a clear relation with cracking tolerance could not be assigned to this gene since high transcript levels were observed in 'Bing' at FS and FC stages (Figure 3F).

\section{CONCLUSIONS}

The results of the present study suggest that differences in the length of fruit growth phases and cracking susceptibility during different stages of fruit development between two contrasting cracking tolerance cultivars could be related to higher levels of transcript detected in genes involved in the synthesis of cuticular waxes like $P a W I N B, P a K C S 6$, and $W S$, and genes related to cell wall modification such as $\beta$-Gal at fruit setting (FS) and fruit color change (FC) stages. The differential expression of wax biosynthesis-related genes in 'Kordia' could provide a continuous wax deposition and a more stable 
membrane that in 'Bing' during the critical first phase of fruit development which, together with the longer time of that growth phase in 'Kordia', could explain the lower levels of cracking index observed in this cultivar. At the same time, high expression levels of $\beta-G a l$ at FS and FR stages can contribute to a greater flexibility and elasticity of the cuticle. Further studies are required to confirm the role of these genes during cherry fruit development, particularly enzymatic activity and cuticle composition analysis between cultivars and fruit developmental stages.

\section{ACKNOWLEDGEMENTS}

This work has been funded by FONDECYT through Grant nr 11100149.

\section{LITERATURE CITED}

Aharoni, A., S. Dixit, R. Jetter, E. Thoenes, G. Van Arkel, and A. Pereira. 2004. The SHINE clade of AP2 domain transcription factors activates wax biosynthesis, alters cuticle properties, and confers drought tolerance when overexpressed in Arabidopsis. Plant Cell 16:2463-2480.

Alkio, M., U. Jonas, T. Sprink, S. Van Nocker, and M. Knoche. 2012. Identification of putative candidate genes involved in cuticle formation in Prunus avium (sweet cherry) fruit. Annals of Botany 110:101-112.

Ambavaram, M.M., A. Krishnan, K.R. Trijatmiko, and A. Pereira. 2011. Coordinated activation of cellulose and repression of lignin biosynthesis pathways in rice. Plant Physiology 155:916-931.

Baggiolini, M. 1952. Stade repères du pecher. Revue Romande d'Agriculture, de Viticulture et d'Arboriculture 4:29-35.

Balbontín, C., H. Ayala, R. Bastias, G. Tapia, M. Ellena, C. Torres, et al. 2013. Cracking in sweet cherries: A comprehensive review from a physiological, molecular and genomic perspective. Chilean Journal of Agricultural Research 73:66-72.

Bastías, R.M., R. Wilckens, A. Venegas, y A. Muñoz. 2010. Dinámica de crecimiento de fruto en cultivares de cerezo (Prunus avium L.) con alta, moderada y baja susceptibilidad a partidura. Agro-Ciencia 26:81-89.

Becker, T., and M. Knoche. 2012. Deposition, strain, and microcracking of the cuticle in developing Riesling' grape berries. Vitis 51:1-6.

Brummell, D.A., and M.H. Harpster. 2001. Cell wall metabolism in fruit softening and quality and its manipulation in transgenic plants. Plant Molecular Biology 47:311-339.

Chang, S., J. Puryear, and J. Cairney. 1993. A simple and efficient method for isolating RNA from pines trees. Plant Molecular Biology 11:113-116.

Christensen, J.V. 1972. Cracking in cherries. III. Determination of cracking susceptibility. Acta Agriculturae Scandinavica 22:128 136.

Christensen, J.V. 2000. Performance in Denmark of 16 European varieties of sweet cherry. Journal of American Pomological Society 54:172-176.

Cosgrove, D. 2000. Expansive growth of plant cell walls. Plant Physiology and Biochemistry 38:109-124.

Hiwasa, K., J. Rose, R. Nakano, A. Inaba, and Y. Kubo. 2003. Differential expression of seven $\alpha$-expansin genes during growth and ripening of pear fruit. Physiologia Plantarum 117:564-572.

Jenks, M., S. Eigenbrode, and B. Lemieux. 2002. Cuticular waxes of Arabidopsis. Journal Cell Biology 17:208-212.

Karaaslan, M., and G. Hrazdina. 2010. Characterization of an expansin gene and its ripening-specific promoter fragments from sour cherry (Prunus cerasus L.) cultivars. Acta Physiologiae Plantarum 32:1073-1084
Kasai, S., H. Hayama, Y. Kashimura, S. Kudo, and Y. Osanai. 2008. Relationship between fruit cracking and expression of the expansin gene MdEXPA3 in 'Fuji' apples (Malus domestica Borkh.) Scientia Horticulturae 116:194-198.

Knoche, M., M. Beyer, S. Peschel, B. Oparlakov, and M.J. Bukovac. 2004. Changes in strain and deposition of cuticle in developing sweet cherry fruit. Physiologia Plantarum 120:667-677.

Knoche, M., B.P. Khanal, and M. Stopar. 2011. Russeting and microcracking of 'Golden Delicious' apple fruit concomitantly decline due to gibberellin A4+7 application. Journal of the American Society for Horticultural Science 136:159-164.

Knoche, M., and S. Peschel. 2006. Water on the surface aggravates microscopic cracking of the sweet cherry fruit cuticle. Journal of the American Society for Horticultural Science 131:192-200.

Kovács, E., Z. Kristóf, R. Perlaki, and D. Szőllősi. 2008. Cell wall metabolism during ripening and storage of nonclimacteric sour cherry (Prunus cerasus L., cv. Kántorjánosi). Acta Alimentaria 37:415-426.

Kunst, L., and A.L. Samuels. 2003. Biosynthesis and secretion of plant cuticular wax. Progress in Lipid Research 42:51-80.

Lane, W., M. Meheriuk, and D. MacKenzie. 2000. Fruit cracking of a susceptible, an intermediate, and a resistant sweet cherry cultivar. HortScience 35:239-242.

Lee, D.H., S.G. Kang, S.G. Suh, and J.K. Byun. 2003. Purification and characterization of $\beta$-galactosidase from peach (Prunus persica). Molecules and Cells 15:68-74.

Li, F., X. Wu, P. Lam, D. Bird, H. Zheng, L. Samuels, et al. 2008 Identification of the wax ester synthase/acyl-coenzyme A: diacylglycerol acyltransferase WSD1 required for stem wax ester biosynthesis in Arabidopsis. Plant Physiology 148:97-107.

Mbéguié-A-Mbéguié, D., B. Gouble, R.M. Gomez, J.M. Audergon, G. Albagnac, and B. Fils-Lycaon. 2002. Two expansin cDNAs from Prunus americana expressed during fruit ripening are differentially regulated by ethylene. Plant Physiology and Biochemistry 40:445-452.

Moctezuma, E., D.L. Smith, and K.C. Gross. 2003. Antisense suppression of a $\beta$-galactosidase gene (TBG6) in tomato increases fruit cracking. Journal of Experimental Botany 54:2025-2033.

Moing, A., C.H. Renaud, H. Christmann, L. Foulihaux, Y. Tauzin, A. Zanetto, et al. 2004. Is there a relation between changes in osmolarity of cherry fruit flesh or skin and fruit cracking susceptibility? Journal of the American Society for Horticultural Science 129:635-641.

Peña, M.J., and N.C. Carpita. 2004. Loss of highly branched arabinans and debranching of rhamnogalacturonan I accompany loss of firm texture and cell separation during prolonged storage of Apple. Plant Physiology 135:1305-1313.

Peschel, S., R. Franke, L. Schreiber, and M. Knoche. 2007. Composition of the cuticle of developing sweet cherry fruit Phytochemistry 68:1017-1025.

Peschel, S., and M. Knoche. 2005. Characterization of microcracks in the cuticle of developing sweet cherry fruit. Journal of the American Society for Horticultural Science 130:487-495.

Prasanna, V., T. Prabha, and R. Tharanathan. 2005. Multiple forms of $\beta$-galactosidase from mango (Mangifera indica L. Alphonso) fruit pulp. Journal of the Science of Food and Agriculture 85:797-803.

Rozen, S., and H.J. Skaletsky. 2000. Primer3 on the WWW for general users and for biologist programmers. p. 365-386. In Krawetz S., and S. Misener (eds.) Bioinformatics methods and protocols: methods in molecular biology. Humana Press, Totowa, New Jersey, USA.

Shi, J.X., S. Malitsky, S. De Oliveira, C. Branigan, R.B. Franke, L. Schreiber, et al. 2011. SHINE transcription factors act redundantly to pattern the archetypal surface of Arabidopsis flower organs. PLoS genetics 7(5):e1001388.

Simon, G. 2006. Review on rain induced fruit cracking of sweet cherries (Prunus avium L.), its causes and the possibilities of prevention. International Journal of Horticultural Science 12:2735 . 
Smith, D.L., J.A. Abbott, and K.C. Gross. 2002. Down-regulation of tomato $\beta$-galactosidase 4 results in decreased fruit softening. Plant Physiology 129:1755-1762.

Vogg, G., S. Fischer, J. Leide, E. Emmanuel, R. Jetter, A. Levy, et al. 2004. Tomato fruit cuticular waxes and their effects on transpiration barrier properties: functional characterization of a mutant deficient in a very-long-chain fatty acid beta-ketoacylCoA synthase. Journal of Experimental Botany 55:1401-1410.

Wakasa, Y., Y. Hatsuyama, A. Takahashi, T. Sato, M. Niizeki, and T. Harada. 2003. Divergent expression of six expansin genes during apple fruit ontogeny. European Journal of Horticultural Science 68:253-259.

Wang, Y., W. Lu, J. Li, and Y. Jiang. 2006. Differential expression of two expansin genes in developing fruit of cracking-susceptible and -resistant litchi cultivars. Journal of the American Society for Horticultural Science 131:118-121.
Yamaguchi, M., I. Sato, A. Watanabe, and M. Ishiguro. 2003. Cultivar differences in exocarp cell growth at apex, equator, stalk cavity and suture during fruit development in sweet cherry (Prunus avium L.) Journal of the Japanese Society for Horticultural Science 72:465-472.

Yeats, T.H., L.B. Martin, H.M. Viart, T. Isaacson, Y. He, L. Zhao, et al. 2012. The identification of cutin synthase: formation of the plant polyester cutin. Nature Chemical Biology 8:609-611.

Yeats, T.H., and J.K.C. Rose. 2008. The biochemistry and biology of extracellular plant lipid-transfer proteins (LTPs). Protein Science 17:191-198.

Zavalloni, C., J.A. Andresen, and J.A. Flore. 2006. Phenological models of flower bud stages and fruit growth of 'Montmorency' sour cherry based on growing degree-day accumulation. Journal of the American Society for Horticultural Science 131:601-607. 